\title{
Increasing the technological process' energy efficiency of the of applying multifunctional wear-and-heat-resistant protective coatings by electro-acoustic spraying
}

\author{
Andrei Kochetov, Elena Fisunova, Tatyana Lavrenova*, and Luybov Filonenko \\ Don State Technical University, Gagarin square, 1, Rostov-on-Don, 344003, Russia
}

\begin{abstract}
The article is devoted to measures improving the energy efficiency of the technological process of applying wear-resistant multifunctional composite protective coatings by the method of electroacoustic spraying. The proposed technique for increasing energy efficiency is based on the use of methods for optimal planning of an experiment using non-orthogonal matrices for planning an experiment and the obtained interpolation-regression equations for the output of the main parameters of the quality of processed products, micro-hardness and roughness, which were tested using the adequacy criterion. The optimization technique based on the criterion of minimizing the reduced energy costs, the technological process of applying wear-resistant protective coatings is based on the determination of the main modes of electro-acoustic spraying according to the specified quality parameters of the required surface layer of the processed mechanical engineering products, micro-hardness and roughness. As a result, from the whole set of solutions of the corresponding equations, we get the opportunity to select those operating modes of the electro-acoustic spraying station that correspond to the minimum values of the amplitude of ultrasonic vibrations and the voltage supplied to the inter-electrode space.
\end{abstract}

\section{Introduction}

The problem of increasing the energy efficiency of the equipment and devices which are used in modern highly efficient technological processes and industries of the mechanical engineering industry is most acute for both blighty and foreign manufacturers of finished products.

Currently, there is a large number of energy-intensive technological processes and industries in many brunches that require urgent intervention and modernization of equipment by using processes and phenomena based on fundamentally new physical foundations and principles. Let us consider in this publication the possibilities of optimizing the unique technological process of applying multifunctional composite protective coatings by electro-

\footnotetext{
* Corresponding author: bys ka87@mail.ru
} 
acoustic spraying. The control of obtaining the predicted physical and mechanical properties of protective coatings of machine parts and shaping tools in a complex acoustic field by the method of electro-acoustic spraying is carried out through the use of highly concentrated sources of electrical energy, which requires an increase in the energy efficiency of equipment in the general context of reducing the reduced energy costs. The prediction of the physical and mechanical properties of the hardened surface of machine parts is carried out on the basis of the method of optimal planning of the experiment using non-orthogonal planning matrices. The adequacy of the obtained interpolation-regression mathematical models was carried out using Fisher's criterion of adequacy. A block diagram of a microprocessor control system for the electro-acoustic spraying process has been developed. The physical foundations of the mass transfer of materials to a substrate in a complex acoustic field, realized by transforming longitudinal vibrations into longitudinal-torsional ones, have been developed. The use of a complex acoustic field formed by a longitudinal-torsional ultrasonic concentrator in this process and high values of the material cooling temperature make it possible to form such elements as inter-metallic compounds, carbides and carbonitrides on the surface of the processed material. The impact produced by an ultrasonic concentrator - an electrode with a shear on the surface, leads to microplastic deformations of the surface layer of the processed material, which provide the required quality parameters of the formed coating and its physical and mechanical properties (microhardness and roughness), while the thickness of the sprayed layer is from 6 to 8 micrometers, and the thickness of the plastically deformed sublayer of the multifunctional composite coating is from 30 to 45 micrometers.

The problem of increasing the energy efficiency of the technological process of synthesizing the predicted physical and mechanical properties and the specified service life of multifunctional composite wear-and-heat protective coatings in the form of barrier layers and an increase in the service life of the shaping tool and mechanical engineering products are inherently intertwined. At the moment, there is a wide variety of the latest highly efficient technological processes, but a special place here should be given to the method of acoustic application of multifunctional protective coatings by the ELAN method. This method is innovative in the area of multifunctional wear and heat resistant protective coatings, which allows the formation of nanocrystalline protective films in absolutely any conductive materials and substrates [1]. This synthesis technology is based on the use of electrical energy of a discharge impulse and a mighty complex acoustic field.

The aim of the work is to optimize the process of obtaining multifunctional wear-andheat-resistant protective coatings based on the mass transfer of the material of the concentrator-electrode and the subsequent synthesis of structures with predicted physical and mechanical properties by the method of electro-acoustic spraying.

To achieve the above goal, a generalized model of the technological process of synthesis of multifunctional wear-and-heat-resistant protective coatings obtained by the method of electro-acoustic deposition of multifunctional protective coatings was developed, a wide range of studies was carried out, which required the use of modules of the software and hardware complex of the autotechnology control system specially developed for these purposes.

\section{Equipment, samples and research methods}

\subsection{Installation of electro-acoustic spraying}

Installation of electro-acoustic spraying of multifunctional composite wear-heat-resistant protective coatings of machine parts is schematically shown in Figure 1. 
The block diagram of the electro-acoustic spraying unit consists of: 1 - an ultrasonic concentrator with an electrode fixed at its end; 2 - processed product; 3 - acoustic feedback sensor; 4 - magnetostrictive transducer (PMS1-1); 5 - ultrasonic generator (UZG 1-1); 6control system; 7- electronic switch; 8 - power supply source; $\mathrm{C}$ - the discharge capacity of the electric energy storage.

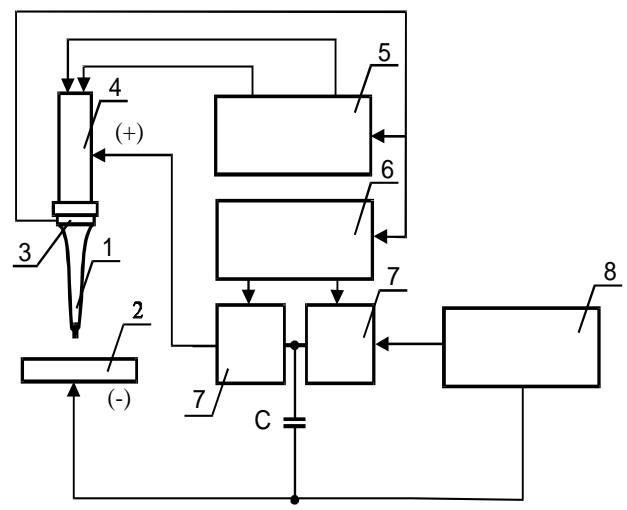

Fig. 1. The block diagram of electro-acoustic spraying

\subsection{Technology for applying multifunctional wear-resistant protective coatings}

A generalized physical model of the process of applying wear-heat-resistant protective coatings when applying multifunctional wear-heat-resistant protective coatings on mechanical engineering products and shaping tools is shown in Figure 2.

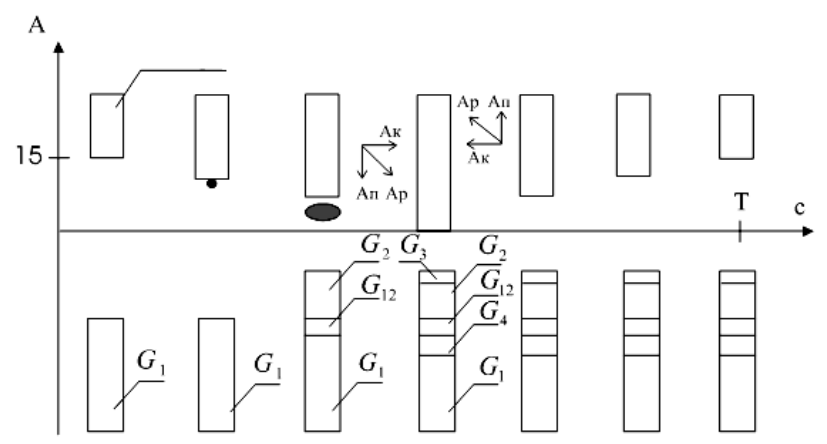

Fig. 2. Model of application of wear-resistant protective coating: $G_{1}, G_{12}, G_{2}, G_{3}, G_{4}$ - material shear modulus; $\bar{A}_{n}$ - vector which, is characterized the longitudinal component of the ultrasonic vibrations of the acoustic system; $\overline{A_{k}}$ - vector, which is characterized the torsional component of the acoustic system; $\bar{A}_{p}$ - resulting vector of complex acoustic field.

An ultrasonic generator (UZG-1-1, power $1 \mathrm{~kW}$ ), to which an acoustic transducer (PMS $1-1$, power $0.9 \mathrm{~kW}$ ) is connected, is the source of ultrasonic energy in the system for applying multifunctional wear-resistant protective coatings. The acoustic concentrator is connected to a magnetostrictive transformer using an acoustic transformer, which forms a 
complex longitudinal-torsional ultrasonic field with an allowed vibration frequency of 22 $\mathrm{kHz}$. A microprocessor-based control system for the generation of discharge pulses interrogates an inductive acoustic feedback sensor, which allows you to determine in which phase the concentrator converter is located, which forms a master action for the power storage of electrical energy, as a result of which, when the concentrator electrode is brought to the surface of the workpiece, the system supplies a optimal shape of the leading and trailing edges. As a result of a highly concentrated electric field, when it is brought into the interelectrode gap of a time-varying potential and complex acoustic vibrations, three phases of the concentrator-electrode arise: liquid, solid, and gaseous. The shape of the discharge pulse and its fronts are optimized according to the criterion of the maximum content of the liquid phase of the electrode material, while ensuring the maximum value of mass transfer.

On the material synthesized as a result of the complex action of highly concentrated energy sources of an electric spark and a complex ultrasonic field, which is in a quasi-liquid phase, under the joint action of kinetic energy and an electric field, it forces to form a channel that carries out mass transfer towards the negative pole of the cathode of the workpiece.

The concentrator-electrode, fixed at the opposite end of the acoustic speed concentrator, due to its design features, performs longitudinal-torsional acoustic vibrations, which have a powerful dispersing effect on the material in the liquid phase. In this case, the active volume of interaction with the environment of the liquid phase sharply increases $V_{0}$ and $N$, $\mathrm{C}, \mathrm{O}_{2}$ and other elements enter into the reaction from the environment.

During the next half-period, the material of the concentrator-electrode material is transferred to the substrate of the material, and the ultra-high values of the cooling temperature and the complex effect of highly concentrated energy sources of an electric spark and a complex longitudinal-torsional ultrasonic field lead to the formation of an amorphous layer on the surface of the article to be hardened, which contains carbides , carbonitrides and intermetallic compounds, imparting special physical and mechanical properties to the synthesized multifunctional protective coating.

After that, the ultrasonic waveguide-concentrator with an electrode fixed at its end makes mechanical contact with shear, which leads to irreversible microplastic deformations of both the multifunctional protective coating itself and the processed substrate material.

At the next stage of the process, the concentrator waveguide is removed from the surface of the sprayed product. Analysis of the synthesized structures as a result of using the method of electro-acoustic deposition shows that, based on the physicomechanical and operational properties of the layers, we can aggregate them into composite layers that prevent the emergence of crystal lattice defects (dislocations) on the material boundary surface. The first composite layer will be formed by barriers with a shear modulus $G_{12}$ and $G_{4}$, second $-G_{2}$ and $G_{3}$ [2].

The presented generalized model of the synthesis of multifunctional composite wearand-heat protective coatings by the method of electro-acoustic spraying makes it possible to clarify the effects of the formation of barriers that prevent both the emergence of crystal lattice defects (dislocations) on the surface and an increase in the service life of agricultural engineering products by suppressing sliding and increasing the microhardness of the surface layer of the workpiece.

The process of applying coatings by electro-acoustic spraying makes it possible to synthesize a multifunctional composite wear-and-heat protective coating, the action of which is based on the mechanism of transfer and implantation of hard alloys [3]. The first protective layer of the multifunctional composite coating is formed due to irreversible microplastic deformation of the near-surface layer of the substrate, and the second - by an amorphous layer of the deposited electrode material. 


\subsection{Experimental data processing technique}

To predict the main parameters of the quality of the processed products of agricultural engineering and to optimize the process of applying multifunctional composite wear-andheat protective coatings according to the criterion of minimizing the reduced costs, the method of planning an experiment with non-orthogonal matrices was used.

As a model, we take a linear model of dependence (1)

$$
\hat{Y}=B_{0}+B_{1} X_{1}+B_{2} X_{2}+\ldots+B_{N} X_{N}+B_{12} X_{1} X_{2}+\ldots+B_{23} X_{2} X_{3} \ldots
$$

To process the obtained experimental data, a specialized software package was developed. The program allows you to approximate linear and nonlinear models.

A matrix of levels of factors $X$ and a matrix of experimental results with $\mathrm{m}, \mathrm{n}, \mathrm{k}$ are set, where $m$ is the number of rows for the matrix of input and output parameters; $n$ is the number of columns of the matrix of input parameters; $k$ is the number of columns of the matrix of output parameters. Regression equation coefficients are estimated using the least squares method.

The calculation of the main parameters is carried out on a computer using the presented software package. In our case, when checking the adequacy of the "roughness" model, we received a negative result, which indicates its inadequacy. Therefore, it is necessary to adopt a new model, which is represented by the expression (2)

$$
\begin{aligned}
Y= & B_{0}+B_{1} X_{1}+B_{2} X_{2}+B_{3} X_{3}+B_{4} X_{4}+B_{5} X_{1} X_{2}+B_{6} X_{1} X_{3}+B_{7} X_{1} X_{4}+ \\
& +B_{8} X_{2} X_{3}+B_{9} X_{2} X_{4}+B_{10} X_{3} X_{4}+B_{11} X_{1}^{2}+B_{12} X_{2}^{2}+B_{13} X_{3}^{2}+B_{14} X_{4}^{2}
\end{aligned}
$$

Let's check the adequacy of the above model. According to Fisher's criterion, we determine the table value $F_{\text {theor }}=2.8$ for level $(0.95)$, and then determine the experimental value $F_{\text {exp. }}=0.431$. The model is adequate since

$$
F_{\text {theor. }} \succ F_{\text {exp. }}
$$

According to the results obtained during a series of experiments devoted to the study of the behavior of the roughness of the obtained surface layer of the hardened samples during electro-acoustic spraying, the model was adopted [4], and also preliminary conclusions can be drawn:

- the complex effect exerted during the hardening of samples by the method of electroacoustic spraying causes a change in the roughness of the hardened surface layer, depending on the modes of its processing;

- the functional dependence of the roughness on the voltage supplied to the electrode, the amplitude of oscillations of the acoustic system, the pressing and feeding forces $R_{a}\left(U_{P}\right.$, $A, P, S)$;

- The strongest effect on the roughness of the resulting surface layer of hardened specimens is exerted by the amplitude of vibrations of the acoustic system and the supply;

- a less strong effect on the roughness of the resulting surface layer of hardened samples is exerted by the voltage applied to the electrode and the clamping force;

- the resulting hardened layer has the same roughness in both the longitudinal and transverse directions.

\section{Discussion of research results}

Using the methodology of optimal planning of the experiment, we needed to establish the dependences of the main operational characteristics of machine parts: microhardness HV (4) and roughness $\mathrm{Ra}$ (5) on the operating modes of the station for electro-acoustic 
deposition of multifunctional protective coatings. As a result, we received the following dependencies:

$$
\begin{aligned}
H V= & \mathbf{5 6 9 9}, 7+3339,6 X_{1}+\mathbf{7 1 1}, \mathbf{8} X_{2}+170,2 X_{3}+\mathbf{3 4 1}, \mathbf{2} X_{4}+\mathbf{3 8 4}, \mathbf{1} X_{1} X_{2}+ \\
& +\mathbf{1 3 5}, 9 X_{1} X_{3}-\mathbf{2 2 4 , 6} X_{1} X_{4}+\mathbf{4 6 , 4} X_{2} X_{3}+\mathbf{2 9 9}, \mathbf{4} X_{2} X_{4}-\mathbf{2 1 0}, 9 X_{3} X_{4}+ \\
& +\mathbf{2 5 2 , 5} X_{1}^{2}-\mathbf{6 8 3}, 7 X_{2}{ }^{2}-\mathbf{6 7 3 , 9} X_{3}{ }^{2}-\mathbf{5 1 4 , 2} X_{4}^{2} \\
R_{a} & =\mathbf{0 , 7 7}+\mathbf{0 , 0 5 8} X_{1}-\mathbf{0 , 0 8 8} X_{2}-\mathbf{0 , 0 3 4} X_{3}-\mathbf{0 , 1 0 5} X_{4}+\mathbf{0 , 0 2 4} X_{1} X_{2}+\mathbf{0 , 0 0 3} X_{1} X_{3}- \\
& -\mathbf{0 , 0 2 8} X_{1} X_{4}+\mathbf{0 , 0 0 4} X_{2} X_{3}+\mathbf{0 , 0 5 0} X_{2} X_{4}+\mathbf{0 , 0 4 2} X_{3} X_{4}+\mathbf{0 , 0 8 9} X_{1}^{2}-\mathbf{0 , 0 5 4} X_{2}{ }^{2}- \\
& -\mathbf{0 , 0 2 2} X_{3}^{2}-\mathbf{0 , 0 2 4} X_{4}{ }^{2}
\end{aligned}
$$

where $X_{1}$ - inter-electrode gap voltage; $X_{2}$ - vibration amplitude of the ultrasonic system circuit; $X_{3}$ - clamping force; $X_{4}$ - product feed.

The given dependences are rather complex, therefore, the use of the obtained data in an analytical form for optimization is very difficult. In addition, the permissible range of variation of the main modes, the so-called working area, imposes additional restrictions on the possibility of obtaining the specified parameters of the surface quality.

Let us set the goal to minimize the values of the voltage $U_{P}$ applied to the electrode and the amplitudes of ultrasonic vibrations $A$ for permissible values of the feed $S$ and a fixed value of the clamping force $\mathrm{P}$, provided that the required parameters of the surface quality are set - microhardness and roughness. For the required value of microhardness, in accordance with expression (4), we construct the dependence of the supply value on the voltage at the electrode $U_{P}$ and the amplitude of ultrasonic vibrations A [5-8].

The numerical solution of this expression with respect to the $\mathrm{x}_{4}$ parameter and further three-dimensional interpolation was performed using a program written in the MATLab environment. The surface shape changes depending on the specified microhardness value, the surface type for the value HV $=7001$ is shown in Figure 3.

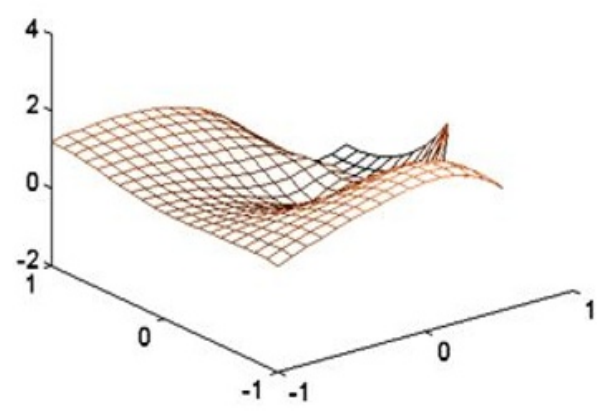

Fig. 3. Surface $X_{4}=f\left(X_{1}, X_{2}\right)$ for $H V=7001$ under $X_{3}=0.5 ; X_{1}-$ voltage applied to the electrode; $X_{2}$ - speaker amplitude; $X_{3}$ - force clamping; $X_{4}$ - feed.

Similarly, it is possible to obtain surfaces for the required value of the roughness parameter, in particular for $\mathrm{Ra}=0.63$ (Figure 4). 


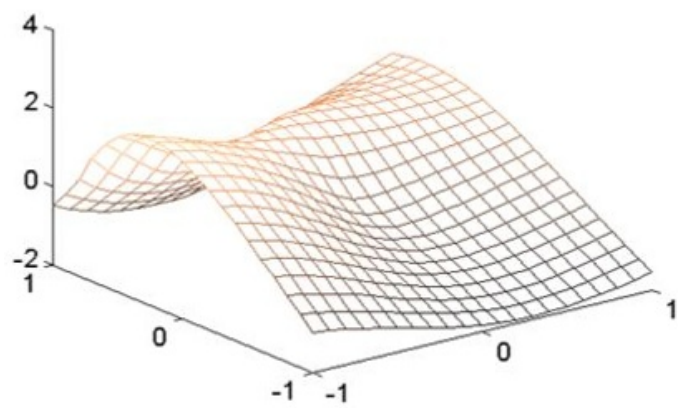

Fig. 4. Surface $X_{4}=f\left(X_{1}, X_{2}\right)$ for $R a=0.63$ under $X_{3}=0.5 ; X_{1}$ - voltage applied to the electrode; $X_{2}-$ speaker amplitude; $X_{3}$ - force clamping; $X_{4}$ - feed.

The graphic interpretation of the corresponding level surfaces is shown in Figure 5.

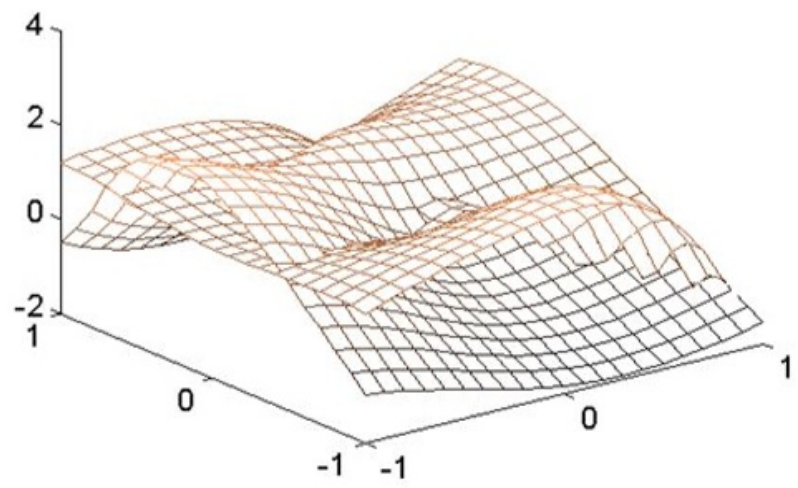

Fig. 5. Intersection of quality parameter surface plots $H V=7001$ and $R a=0.63$.
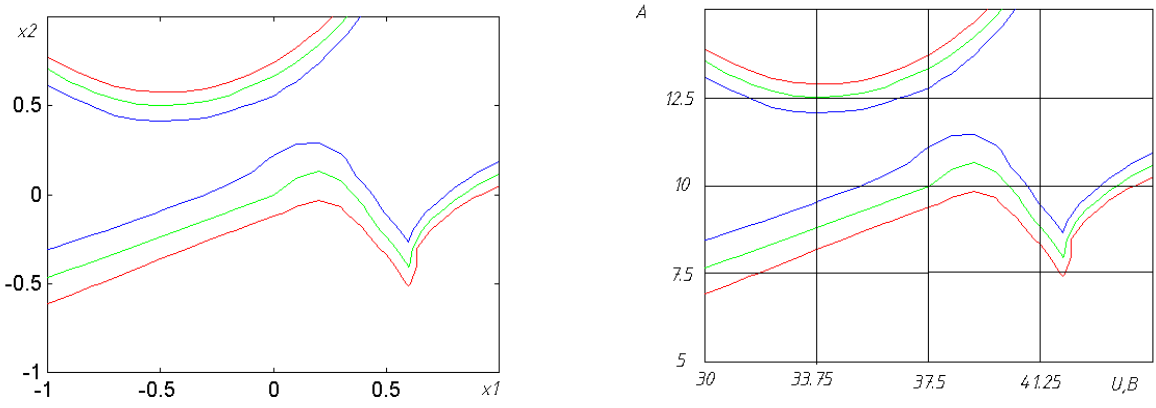

Fig. 6. Projection of the set of solutions to a system of equations $X_{4}=f\left(x_{1}, x_{2}\right)$ for the given quality parameters $H V=7000$ and $R a=0.63$ on the plane $x_{I} x_{2}$. 

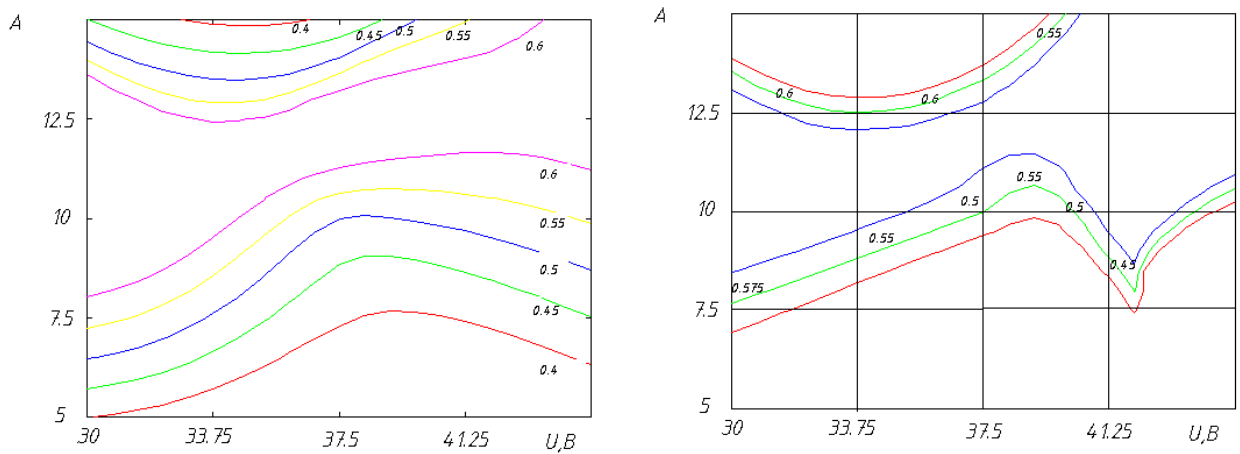

Fig. 7. Level surfaces for different feed rates $x_{4}$.

The given graphic interpretations represent a set of solutions of equations (4) and (5) with respect to the feed rate $x_{4}$ at a fixed value of the clamping force $x_{3}$ in the range of admissible values of the inter-electrode gap voltage $x_{1}$ and the amplitude of the values of acoustic vibrations $x_{2}$. The joint solution of the previously presented equations is the intersection of the graphs of the surfaces corresponding to each of the equations (Figure 5).

The projection of the set of solutions to the system of equations on the $x_{1} x_{2}$ plane, shown in Figure 6, allows one to draw conclusions about possible implementations of product processing modes and choose from them the mode that corresponds to the minimum values of energy costs [9-11]. Determination of the value of the value of the electrode supply relative to the movement of the product is carried out according to the nomograms, which are presented in Figure 7.

The obtained graphical interpretations of the dependences of microhardness and roughness on the voltage supplied to the electrode, the amplitude of the ultrasonic system oscillations, the pressing and feeding forces $R_{a}\left(U_{P}, A, P, S\right)$ using the methods of mathematical planning of the experiment allows taking into account simultaneously several main factors that determine the mode of electro-acoustic spraying., such as: the voltage applied to the electrode, the amplitude of ultrasonic vibrations, the amount of supply which is undoubtedly a promising direction in the search for optimal modes.

A technique has been developed for the accelerated computational and analytical selection of the optimal parameters of the electro-acoustic spraying mode on the basis of multifactor planning of the experiment, which made it possible to optimize the time for calculating the main physical and mechanical properties of the processed products. The result of solving the presented problem in the form of a software module of predicted tribological and operational properties makes it possible to obtain nomograms of the main modes depending on the specified parameters of the surface quality [12].

Analyzing the obtained experimental data and proceeding from the need of mechanical engineering in obtaining high-quality multifunctional wear-and-heat-resistant protective coatings, it is possible to give recommendations for setting an energy-efficient mode: voltage supplied to the electrode $U_{P}=37.5 \mathrm{~V}$, amplitude of ultrasonic circuit oscillations A $=10 \mu \mathrm{m}$, feed $0.5 \mathrm{~mm} / \mathrm{min}$, force clamping $S=12.5 \mathrm{H}$.

Based on the verification of the adequacy of the obtained mathematical models using the Fisher criterion and the method of analysis of residues, we can assert that they can be used in the proposed optimization technique, according to the criterion of the reduced energy efficiency of the process of applying multifunctional wear-resistant protective coatings by electro-acoustic spraying [7]. At the first step, it is necessary to obtain level surfaces for the required microhardness $\mathrm{HV}=7000$ according to Vickers and roughness 
$\mathrm{Ra}=0.63 \mu \mathrm{m}$. At the second step, we carry out a joint solution by graphically intersecting the corresponding surfaces shown in Figure 3. At the third step, we determine the set of solutions (points of intersection of surfaces), from which we select those values that will correspond to the minimum values of the amplitude of ultrasonic vibrations and the voltage supplied to the working bodies stations for applying wear-resistant protective coatings of mechanical engineering products and shaping tools, which will dramatically increase the energy efficiency of this technological process.

The given dependences are rather complicated, therefore, the use of the obtained data in an analytical form to increase the energy efficiency of this technological process is very difficult in the real sector of production, and the permissible range of variation of the main modes, the so-called working area, imposes additional restrictions on the possibility of obtaining the specified parameters of the surface quality [ 13]. Therefore, the proposed optimization method should be automated at the level of the hardware-software complex of the microprocessor control system, based on the creation of specialized maps for the used materials of substrates and electrodes.

\section{Conclusion and general findings}

On the basis of theoretical and experimental studies, an autotechnologist module of the automated control system of the electro-acoustic spraying station of multifunctional wearand-heat protective coatings of machine parts and shaping tools was developed. A structural diagram has been developed, a generalized model and a technique for optimizing the process of applying a wear-and-heat protective coating of machine parts and general engineering products, a shaping tool working in severe operating conditions [14], based on the criterion of minimizing energy costs, has been proposed. The error of the obtained mathematical models of the dependences of microhardness and roughness on the main spraying modes does not exceed 5\%, which indicates a good convergence of the results. The proposed optimization method according to the criterion of the reduced costs will allow not only to reduce the consumption of electrical energy of the technological process, but also to increase the service life of the executive equipment of the station for electroacoustic deposition of wear-resistant protective coatings, which ultimately will reduce the operating costs by up to $20 \%$. The versatility of the method, the low cost of applying multifunctional coatings, and the ease of implementation with the possibility of utilizing hard metal waste make this technology economically attractive in comparison with existing methods and technologies [15]. The technology of applying multifunctional wear-andheat-resistant protective coatings by electro-acoustic spraying in a complex ultrasonic field, implemented by means of a longitudinal-torsional concentrator, makes it possible to realize the effect of a variable vector of acoustic vibrations during the formation of the rheological properties of conductive multifunctional wear-and-heat protective coatings with an amorphous structure, which gives the complex unique performance properties.

\section{References}

1. Mashhour A. Alazwari, Singiresu S. Rao, Composites Part B: Engineering, 161, 107120, (2019)

2. Jitendra M. Mistry, Piyush P. Gohil, Composites Part B: Engineering, 161, 190-204, (2019)

3. Yingwu Zhou, Yaowei Zheng, Jun Pan, Lili Sui, Pengda Li, Composites Part B: Engineering, 161, 272-284, (2019) 
4. Jianwu Zhou, Binbin Liao, Yaoyao Shi, Yangjie Zuo, Liyong Jia, Composites Part B: Engineering, 161, 300-313, (2019)

5. Longchao Zhuo, Zhao Zhao, Zhicong Qin, Qiuyu Chen, Feng Wang, Composites Part B: Engineering, 161, 336-343, (2019)

6. Giap X. Ha, Dragan Marinkovic, Manfred W. Zehn, Composites Part B: Engineering, 161, 427-438, 2019

7. Lei Guo, Zheng Yan, Xue Dong, Xichun Liu, Fuxing Ye, Composites Part B: Engineering, 161, 473-482, (2019)

8. Jianjun Wang, Lihua Wen, Jinyou Xiao, Tengfei Liang, Penghui Li, Composites Part B:Engineering, 161, 502-513, (2019)

9. Dhanasekaran Vikraman, K. Karuppasamy, Sajjad Hussain, A. Kathalingam, HyunSeok Kim, Composites Part B: Engineering, 161, 555-563, (2019)

10. Worajak Janwaen, Joaquim A. Barros, Inês G. Costa, Composites Part B: Engineering, 158, 67-81, (2019)

11. V. L. Gaponov, D. M. Kuznetsov, V. V. Dudnik, N. P. Shabelskaya. Bulletin of the Technological University. 23, 7. 28-32, (2020)

12. Kuznetsov D. M., Gaponov V. L., Gaidukova Yu. A., Maslova E. E. Modern hightech technologies. 4, 74-79, (2018)

13. Builo, S.I., Kuznetsov, D.M., Gaponov, V.L, Trepachev, V.V. Russian Journal of Nondestructiv Testing, 48, 10, 594-597, (2012)

14. Builo, S.I.; Kuznetsov, D.M.; Gaponov, V.L. and Balakai, V.I. The Acoustic Emission Diagnostics of Galvanic Process Kinetics. Chapter 13 (pp. 457-462). In: Proceedings of the 2016 International Conference on Physics and Mechanics of New Materials and Their Applications, Ivan A. Parinov, Shun-Hsyung, Muaffaq A. Jani (Eds.). Nova Science Publishers, New York (2017)

15. Gaponov V, Kuznetsov D, Dudnik V, Afanasieva N MATEC Web Conferences 117, 7, (2017) 